\title{
Methodology for vetting heavily doped semiconductors for intermediate band photovoltaics: A case study in sulfur-hyperdoped silicon
}

\section{Citation}

Sullivan, J. T., C. B. Simmons, J. J. Krich, A. J. Akey, D. Recht, M. J. Aziz, and T. Buonassisi. 2013. "Methodology for Vetting Heavily Doped Semiconductors for Intermediate Band Photovoltaics: A Case Study in Sulfur-Hyperdoped Silicon." Journal of Applied Physics 114 (10): 103701. doi:10.1063/1.4820454.

\section{Published Version}

doi:10.1063/1.4820454

\section{Permanent link}

http://nrs.harvard.edu/urn-3:HUL.InstRepos:14550025

\section{Terms of Use}

This article was downloaded from Harvard University's DASH repository, and is made available under the terms and conditions applicable to Other Posted Material, as set forth at http:// nrs.harvard.edu/urn-3:HUL.InstRepos:dash.current.terms-of-use\#LAA

\section{Share Your Story}

The Harvard community has made this article openly available.

Please share how this access benefits you. Submit a story. 


\title{
Methodology for vetting heavily doped semiconductors for intermediate band photovoltaics: A case study in sulfur-hyperdoped silicon
}

\author{
J. T. Sullivan, ${ }^{1, \text { a) }}$ C. B. Simmons, ${ }^{1}$ J. J. Krich, ${ }^{2}$ A. J. Akey, ${ }^{3}$ D. Recht, ${ }^{3}$ M. J. Aziz, ${ }^{3}$ \\ and T. Buonassisi ${ }^{1}$ \\ ${ }^{1}$ Massachusetts Institute of Technology, Cambridge, Massachusetts 02139, USA \\ ${ }^{2}$ University of Ottawa, Ottawa, Ontario K1N 6N5, Canada \\ ${ }^{3}$ Harvard School of Engineering and Applied Sciences, Cambridge, Massachusetts 02138, USA
}

(Received 29 May 2013; accepted 20 August 2013; published online 9 September 2013)

\begin{abstract}
We present a methodology for estimating the efficiency potential for candidate impurity-band photovoltaic materials from empirical measurements. This methodology employs both Fourier transform infrared spectroscopy and low-temperature photoconductivity to calculate a "performance figure of merit" and to determine both the position and bandwidth of the impurity band. We evaluate a candidate impurity-band material, silicon hyperdoped with sulfur; we find that the figure of merit is more than one order of magnitude too low for photovoltaic devices that exceed the thermodynamic efficiency limit for single band gap materials. (C 2013 AIP Publishing LLC. [http://dx.doi.org/10.1063/1.4820454]
\end{abstract}

\section{INTRODUCTION}

Intermediate-band (IB) solar cells are a proposed pathway to exceed the Shockley-Queisser ${ }^{1}$ efficiency limit. ${ }^{2-5}$ The introduction of an additional, intermediate band of electronic levels within the band gap could dramatically increase device current through a two-photon process while only resulting in small losses in operating voltage. ${ }^{6}$ Theoretical investigations $^{2,7,8}$ indicate that intermediate-band solar cells can achieve efficiencies over $60 \%$, though no intermediate-band devices have been demonstrated with efficiencies exceeding the Shockley-Queisser limit. Experimental work has primarily focused on three material systems: highly mismatched alloys, ${ }^{9-14}$ quantum dot structures, ${ }^{15-17}$ and impurity-band materials. ${ }^{3,4,18}$ In this article, we will focus on the latter concept, which has been studied extensively from a materials standpoint in two primary material systems: silicon hyperdoped with $\mathrm{Ti}(\mathrm{Si}: \mathrm{Ti})^{19-26}$ and silicon hyperdoped with sulfur (Si:S). ${ }^{27-35}$ Despite significant efforts on these impurity-band materials, no high-efficiency devices have been demonstrated. We present an experimental framework that predicts whether a candidate impurity-band material system will actually enhance the efficiency of a photovoltaic (PV) device.

The standard architecture for an intermediate-band solar cell is a $p$-IB- $n$ device. ${ }^{3}$ However, an intermediate-band material must meet certain requirements to demonstrate an efficiency enhancement over a traditional pn junction device. ${ }^{36}$ First, it must possess the appropriate band structure-i.e., an IB that is separate from both the conduction band (CB) and the valence band $(\mathrm{VB}){ }^{37}$ It must be able to create both excess free electrons and holes using a two-photon process where both photons have energies lower than the CB-VB band gap (we refer to these photon energies as "sub-band gap"). ${ }^{38}$ And lastly, it must have both sufficiently good optical and transport properties so that it can create mobile charges using sub-band gap light and subsequently transport these charges to the neighboring $p$ or $n$ regions. ${ }^{36}$ If the

a) jts48@cornell.edu material fails to meet any of these three requirements, then it cannot boost the efficiency of a $p n$-junction solar-cell device.

The non-dimensional figure of merit $\nu$ provides a quantitative evaluation of whether an IB material has the potential to enhance the efficiency of a solar device. ${ }^{36}$ It is defined as the ratio of the recombination lifetime for photogenerated carriers $\tau$ to the transit time $t$ for these carriers to exit the IB material in a $p$-IB- $n$ device structure. For a planar, driftdriven device, ${ }^{39} t$ is related to the mobility $\mu$, built-in voltage $V_{b i}$, and IB layer thickness $w$ by $t=w^{2} /\left(\mu V_{\mathrm{bi}}\right)$. The IB layer must absorb the majority of the incident sub-band gap light, so it needs to have a thickness on the order of the absorption length, thus, $w=c / \alpha$, where $c$ defines the number of absorption lengths and $\alpha$ represents the optical absorption coefficient for sub-band gap photons. (It has been suggested that a value of $c=2-3$ is sufficient. ${ }^{36}$ ) The result for $\nu$ is

$$
\nu=\frac{1}{c^{2}} V_{\mathrm{bi}} \mu \tau \alpha^{2} .
$$

This figure of merit encompasses the inherent tradeoff in the thickness of the IB layer; a thicker layer will absorb a larger fraction of sub-band gap light, but for a given carrier lifetime $\tau$, a thicker layer reduces the fraction of photogenerated carriers that are extracted prior to recombination. For an IBPV device to have a higher efficiency than a single junction device, the IB layer must have $\nu \geq 1 .{ }^{36}$ In principle, IB materials have different figures of merit for both holes and electrons and both must be large. Herein, we focus on the electron figure of merit, $\nu_{e}=c^{-2} V_{\mathrm{bi}} \mu_{\mathrm{e}} \tau_{\mathrm{e}} \alpha_{\mathrm{e}}^{2}$.

In this paper, we evaluate the figure of merit for a candidate impurity-band material, Si:S. Sulfur is known to produce deep levels within the silicon band gap. ${ }^{40}$ Based on optical and transport data, $\mathrm{Si}: \mathrm{S}$ is a candidate material for an IB absorber layer. At 0.5 at. $\% \mathrm{~S}$ concentration, this material exhibits strong sub-band gap absorption. ${ }^{30,41}$ Photodiodes made from S-doped $\mathrm{Si}$ have exhibited enhanced photoresponse in the infrared (IR). ${ }^{42}$ Additionally, metallic conductivity at very high $\mathrm{S}$ concentrations has been observed, an 
indication of the formation of a dopant-induced metallic band. ${ }^{43}$ We study three samples with a range of sulfur concentrations, including one with metallic conductivity.

Each element of $\nu$ can be measured or estimated in a material without the need to fabricate a solar-cell device. For example, $V_{b i}$ depends on the doping profile in a device, but is generally smaller than the band gap of the material. ${ }^{44}$ Here, we determine $\nu_{\mathrm{e}}$ for Si:S by taking $c=2, V_{\mathrm{bi}}=E_{\mathrm{g}} / q=1.1 \mathrm{~V}$, and we experimentally measure $\alpha_{\mathrm{e}}$ and $\mu_{\mathrm{e}} \tau_{\mathrm{e}}$.

We measure $\alpha_{\mathrm{e}}$ for sub-band gap photon energies using optical absorption measurements. The absorption spectra serve two purposes. First, the measurements provide $\alpha_{\mathrm{e}}$, which is used to compute $\nu_{\mathrm{e}}$. Second, spectral fits to $\alpha_{\mathrm{e}}$ determine the energetic position and width of the S-induced impurity states, which is used to reject materials that lack the desired band structure (i.e., the materials with $\mathrm{S}$ concentrations so large that the impurity band is degenerate or neardegenerate with the $\mathrm{CB}$ ).

We determine $\mu_{e} \tau_{e}$ by low-temperature photoconductivity measurements. Previous room-temperature photoconductivity measurements of $\mathrm{Si}: \mathrm{S}$ did not produce detectable photoresponse, ${ }^{33}$ allowing researchers to place an upper limit on $\mu_{e} \tau_{e}$ of $1 \times 10^{-7} \mathrm{~cm}^{2} / \mathrm{V}$, based on the noise floor of the measurement. ${ }^{29}$ However, this value of $\mu_{\mathrm{e}} \tau_{\mathrm{e}}$ is consistent with $\nu_{\mathrm{e}}$ greater than or less than 1 for $\mathrm{Si}: \mathrm{S}$. Thus, it is not possible to draw a conclusion about the efficiency potential of $\mathrm{Si}: \mathrm{S}$ from this previous bound. We perform photoconductivity measurements at low-temperature to increase the measurement sensitivity by decreasing the background conductivity of the material. We expect $\mu_{\mathrm{e}} \tau_{\mathrm{e}}$ to decrease at higher temperatures, so the low-temperature measurements provide an upper bound on $\mu_{\mathrm{e}} \tau_{\mathrm{e}}$ at room temperature. By combining the values of $\alpha_{\mathrm{e}}$ and $\mu_{\mathrm{e}} \tau_{\mathrm{e}}$ found here, we are able to place a definitive upper bound on $\nu_{\mathrm{e}}$ for Si:S. Additionally, the photoconductivity measurements provide information about the recombination statistics and filling fraction of sulfur trap states in $\mathrm{Si}: \mathrm{S}$, as described in Sec. V.

\section{OPTICAL ABSORPTION MEASUREMENTS}

\section{A. Experimental methods}

Samples for absorption measurements were fabricated using $700 \mu \mathrm{m}$ thick, boron-doped, p-type Si wafers $(10-20 \Omega$-cm, double-side polished, Czochralski-grown, and $\langle 100\rangle$ orientation) that were ion implanted at $95 \mathrm{keV}$ with ${ }^{32} \mathrm{~S}^{+}$at $7^{\circ}$ off normal incidence to prevent channeling effects. Wafers were prepared with three different sulfur doses: $3 \times 10^{15}, 6 \times 10^{15}$, and $1 \times 10^{16}$ atoms $\mathrm{cm}^{-2}$. Implanted wafers were pulsedlaser melted $\left(\mathrm{PLM}^{45}\right)$ to restore crystallinity with four consecutive $\mathrm{XeCl}$ excimer laser pulses ( $308 \mathrm{~nm}$ wavelength, $25 \mathrm{~ns}$ pulse duration full width at half maximum, $50 \mathrm{~ns}$ total duration, and fluences of $1.7,1.7,1.7$, and $1.8 \mathrm{~J} / \mathrm{cm}^{2}$, respectively.) The beam was homogenized to $<4 \%$ root-mean square intensity variation over a $2 \times 2 \mathrm{~mm}^{2}$ area. The resulting material is single-crystal, super-saturated with $\mathrm{S}$ at concentrations orders of magnitude beyond the equilibrium solid-solubility limit, and free of extended structural defects. Further details on the PLM and implantation process can be found in the previous literature. ${ }^{45}$
Sulfur concentration profiles were measured by secondary ion mass spectrometry (SIMS) and found to have peak concentrations of $1.2 \times 10^{20}, 2.1 \times 10^{20}$, and $3.8 \times 10^{20}$ atoms $\mathrm{cm}^{-3}$ for identically prepared samples that received implant doses of $3 \times 10^{15}, 6 \times 10^{15}$, and $1 \times 10^{16} \mathrm{~S} \mathrm{~cm}^{-2}$, respectively. ${ }^{27,43}$ The first $200 \mathrm{~nm}$ from the surface have a nearly constant $S$ concentration, and the next $150 \mathrm{~nm}$ contain a smoothly decreasing S concentration. ${ }^{46}$ Over $95 \%$ of $\mathrm{S}$ dopant is found within the top $350 \mathrm{~nm}$ of the sample.

Both transmission and reflection were measured using Fourier-transform infrared spectroscopy (FTIR) from wavelengths of 1.4 to $16 \mu \mathrm{m}$. An unprotected gold mirror was used as a reflectance standard. Measurements with wavelengths greater than $4.4 \mu \mathrm{m}$ were complicated by vibrational modes in the silicon substrate and water vapor surrounding the samples and are not reported. To decouple the absorption contributions from the S-doped layer and the bulk silicon substrate, a reference sample consisting of the same silicon wafer but without the S-implanted layer was used to determine the complex index of refraction of the substrate.

\section{B. Experimental results}

The absorption coefficient of the hyperdoped sulfur layer was extracted from measured transmission and reflection spectra by modeling the sample as a thin film atop an optically thick substrate surrounded by air. ${ }^{46}$ The absorption coefficients for three samples containing different sulfur concentrations are given in Fig. 1. These results are consistent with previously reported ellipsometry measurements, ${ }^{47}$ but the data reported here extend the range of $\alpha$ to lower photon energies.

Fits to the absorption spectra yield insight into how the band structure changes as a function of sulfur concentration. At low concentrations, S substitutional defects are deep

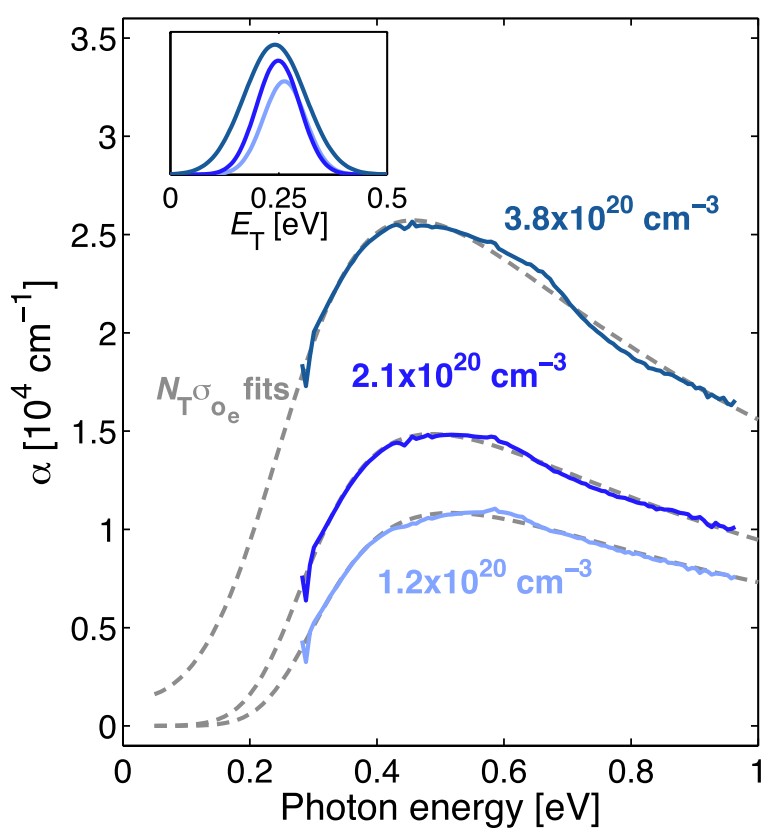

FIG. 1. Absorption coefficient (with free carrier absorption removed) for three different concentrations of sulfur, fit using Eq. (4). The inset shows the probability distribution of trap energies $E_{\mathrm{T}}$ determined by the spectral fits. 
donors. We model the sub-band gap absorption as the excitation of an electron from a $S$ trap state to the conduction band (assumed to be parabolic) and hence we use subscript "e" for the absorption coefficient, $\alpha_{\mathrm{e}}$. We assume that the VB to IB transition is negligibly small (i.e., $\alpha_{\mathrm{e}} \gg \alpha_{\mathrm{h}}$ ), an assumption that is supported by density functional theory calculations of S-doped Si that found the optical transition from VB to IB to be negligible compared to the IB to CB transition. ${ }^{48}$ The modeled absorption coefficient for sub-band gap light is assumed to be a product of the concentration of traps $N_{\mathrm{T}}$ and their optical cross-section $\sigma_{\mathrm{o}_{\mathrm{e}}}$

$$
\alpha_{\mathrm{e}}=N_{\mathrm{T}} \sigma_{\mathrm{o}_{\mathrm{e}}} .
$$

A forbidden vertical transition model ${ }^{49,50}$ for $\sigma_{\mathrm{o}_{\mathrm{e}}}$ gives the best fits to the experimental data, superior to other theoretical models describing optical cross-sections for photoionization of deep-level impurities. ${ }^{50}$ Additionally, the forbidden vertical transition model has previously been used to describe the optical cross-section of $\mathrm{S}$ in $\mathrm{Si}$ at dilute concentrations, ${ }^{51}$ as well as other deep-level impurities in $\mathrm{Si}^{52}$ The model predicts

$$
\sigma_{\mathrm{o}_{\mathrm{e}}} \propto \frac{\left(\hbar \omega-E_{\mathrm{T}}\right)^{3 / 2}}{(\hbar \omega)^{3}},
$$

where $E_{\mathrm{T}}$ is the trap energy and $\hbar \omega$ is the photon energy. Equation (3) describes the excitation from a single discrete trap level. To account for broadening of the trap levels into an impurity band of finite width at the high $\mathrm{S}$ concentrations used here, we convolve Eq. (3) with a Gaussian distribution of trap energies, with a mean trap energy $E_{\mathrm{T}}$ and a standard deviation $E_{\sigma}$

$$
\sigma_{\mathrm{o}_{\mathrm{e}}} \propto \frac{1}{E_{\sigma} \sqrt{2 \pi}} \int_{0}^{\infty} \frac{(\hbar \omega-E)^{3 / 2}}{(\hbar \omega)^{3}} \exp \left[-\frac{\left(E-E_{\mathrm{T}}\right)^{2}}{2 E_{\sigma}^{2}}\right] d E .
$$

A Gaussian distribution was used because it resembles the shape of the mid-gap density of states calculated by density functional theory in an analogous material system, Se hyperdoped silicon (Si:Se). ${ }^{31}$

The spectral fits to $\alpha_{\mathrm{e}}$ and the fit parameters are shown in Fig. 1 and Table I, respectively. The spectral fits in Fig. 1 are created using Eq. (4) with the values reported in Table I. The probability distributions of trap energies for each $\mathrm{S}$ concentration as determined by the spectral fits are plotted in the inset of Fig. 1. For the sample with the highest S concentration, the tail of the trap distribution is degenerate or neardegenerate with the conduction band. This observation is

TABLE I. Fit parameters with $95 \%$ confidence intervals determined by fitting Eq. (4) to absorption coefficient data in Fig. 1.

\begin{tabular}{lccc}
\hline \hline $\begin{array}{l}{ }^{32} \mathrm{~S}^{+} \text {implantation } \\
\text { dose }\left[\mathrm{cm}^{-2}\right]\end{array}$ & Peak S conc. $\left[\mathrm{cm}^{-3}\right]$ & $E_{\mathrm{T}}[\mathrm{meV}]$ & $E_{\sigma}[\mathrm{meV}]$ \\
\hline $3 \times 10^{15}$ & $1.2 \times 10^{20}$ & $263 \pm 2$ & $49 \pm 4$ \\
$6 \times 10^{15}$ & $2.1 \times 10^{20}$ & $249 \pm 2$ & $50 \pm 4$ \\
$1 \times 10^{16}$ & $3.8 \times 10^{20}$ & $241 \pm 2$ & $71 \pm 4$ \\
\hline
\end{tabular}

consistent with the previous reports indicating a merging of the impurity and conduction bands in chalcogen hyperdoped $\mathrm{Si}^{31}$ Additionally, the $\mathrm{S}$ concentration at which this effect is observed corresponds to that which has previously been associated with an insulator-to-metal transition (IMT), ${ }^{43}$ and with a discrete change in electronic structure observed in X-ray emission spectroscopy. ${ }^{30}$ The small optical gap exhibited for the higher $\mathrm{S}$ concentration samples means that this material is unsuitable as an IBPV absorber layer, which requires that the IB be energetically separated from both band edges. ${ }^{37}$ For the remainder of the manuscript, we consider only the lowest $\mathrm{S}$ concentration listed in Table I.

It is important to note that the increase in parameter $E_{\sigma}$ with increased S concentration (see Eq. (4)) could also be due to an increase in electron-phonon coupling, and is mathematically described by replacing $E_{\sigma}^{2}$ with $2 k T d_{\mathrm{FC}}$, where $d_{\mathrm{FC}}$ is the Franck-Condon parameter. ${ }^{53,54}$ However, it seems unlikely that electron-phonon interactions are the major driving force behind the broadening. Previous spectrally resolved temperature-dependent photoconductivity measurements performed under bias (where $\mathrm{S}$ atoms were ionized with $E_{\mathrm{T}} \approx 0.6 \mathrm{eV}$ ) and in short-circuit mode ( $\mathrm{S}$ atoms were neutral with $E_{\mathrm{T}} \approx 0.3 \mathrm{eV}$ ) indicated that spectral broadening due to electron-phonon coupling was strong in ionized $\mathrm{S}$ impurities, ${ }^{55,56}$ but negligible in neutral $\mathrm{S}$ impurities. ${ }^{55}$ Additionally, analysis in Sec. V indicates that the majority $(>90 \%)$ of the trap states are indeed occupied, so the $\mathrm{S}$ atoms are assumed neutral; this is also confirmed by Halleffect measurements that demonstrate low ionization ratios $(<7 \%)$ at room temperature. ${ }^{57}$ Furthermore, since the broadening in our absorption spectra is well-explained by trap states with energies near the neutral S energy $\left(E_{\mathrm{T}} \approx 0.3 \mathrm{eV}\right)$, we attribute the broadening to a concentration-dependent variation in the trap state energies. Also, we neglect any optical transitions from filled to empty states within the IB because the limited bandwidth of that band would not allow such transitions with photon energies greater than $0.3 \mathrm{eV}$.

\section{LOW-TEMPERATURE PHOTOCONDUCTIVITY MEASUREMENTS}

\section{A. Experimental methods}

The relatively high absorption coefficient exhibited by $\mathrm{Si}: \mathrm{S}$ for sub-band gap photon energies makes it a candidate impurity-band material; however, for a high efficiency IBPV device, it must be possible to extract the photogenerated carriers from the IB layer. We measured low-temperature photoconductivity to determine the $\mu_{\mathrm{e}} \tau_{\mathrm{e}}$ product for carriers photo-excited using sub-band gap light. The samples used for the photoconductivity measurements were fabricated in an identical manner to those used for the optical absorption measurements, but with the addition of metallic top contacts and local phosphorous $n$-doping to achieve negligibly small contact resistance (see inset of Figs. 2 and 3 for sample geometry and regions of local doping).

The measurements were performed at a temperature of $36 \mathrm{~K}$ to freeze out the $\mathrm{S}$-donor electrons and to reduce the background conductivity of the sample. Heavy doping of $\mathrm{P}$ locally underneath the metal contacts ensures that contacts 


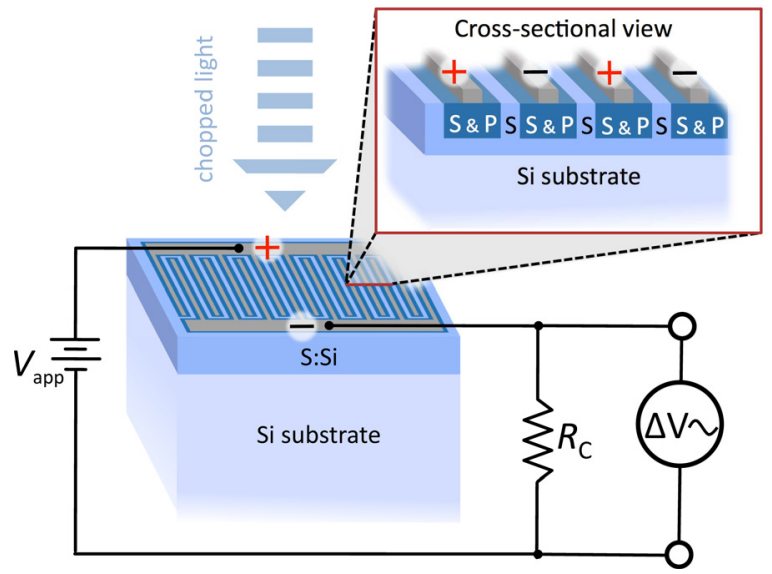

FIG. 2. Sample geometry and the photoconductivity experimental setup for high-chopping frequencies. A DC bias $V_{\text {app }}$ is applied in series across the sample and a comparative resistor of known resistance, $R_{\mathrm{C}}$. The sample resistance varies under the chopped laser illumination. The amplitude of the AC voltage generated across the comparative resistor, $\Delta V$, is measured by a lock-in amplifier. In the top right, a cross sectional view of the contact region illustrates the local phosphorus doping under the metal contacts. Note that the vertical dimension is not drawn to scale.

remain Ohmic at low temperature. Excellent metalsemiconductor contact is vital for two reasons. First, it ensures that the dominant resistance in the sample is the S-doped silicon rather than the contact resistance or the regions doped with $\mathrm{S}$ and $\mathrm{P}$, which enables accurate measurement of the $\mathrm{Si}: \mathrm{S}$ sheet conductivity. Second, heavy doping reduces the possibility of photo-response false positives, such as internal photoemission. ${ }^{58}$

\section{contact control sample}

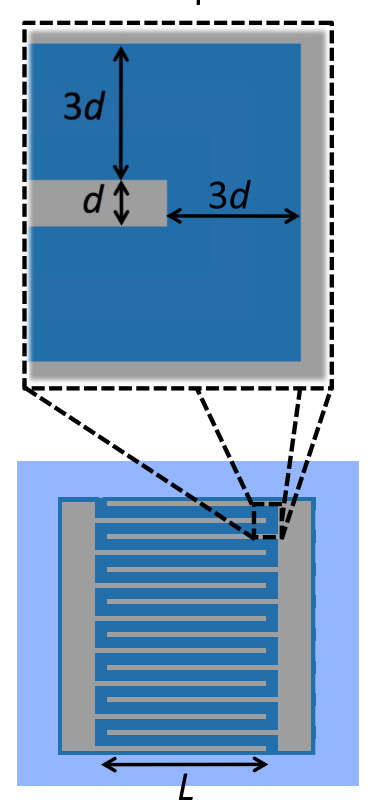

photoconductivity sample

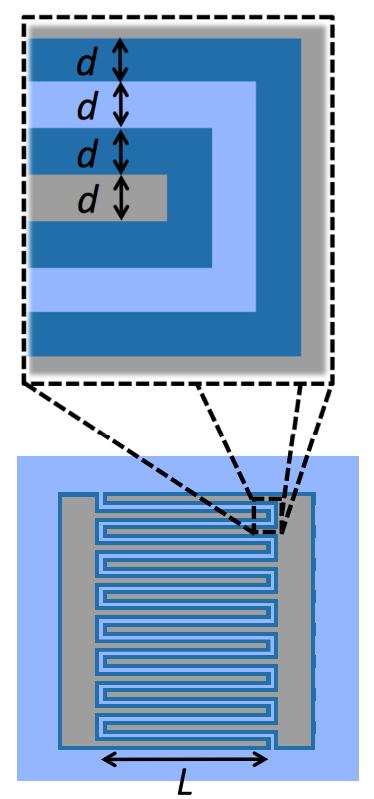

FIG. 3. Top-down view of regions for ion implantation and metal deposition for the contact control sample and photoconductivity sample. Regions in grey show where a $\mathrm{Ti} / \mathrm{Pd} / \mathrm{Ag}$ metal stack was deposited, regions in dark blue were implanted with both $\mathrm{S}$ and $\mathrm{P}$, and regions in light blue were implanted with only $\mathrm{S}$. The feature size $d$ is $25 \mu \mathrm{m}$ and the metal finger length $L$ is $1000 \mu \mathrm{m}$. The number of fingers protruding from each busbar $N_{\mathrm{f}}$ is 8 .
Photoconductivity samples received a blanket ${ }^{32} \mathrm{~S}^{+}$ ion implantation dose of $3 \times 10^{15}$ atoms $\mathrm{cm}^{-2}$ and a second, local ${ }^{31} \mathrm{P}^{+}$ion implantation at $10 \mathrm{keV}$ to a dose of $1 \times 10^{15}$ atoms $\mathrm{cm}^{-2}$. The sample area that received the $\mathrm{P}$ implant was defined by photolithography. An additional control sample received a blanket implant of both the ${ }^{32} \mathrm{~S}^{+}$and the ${ }^{31} \mathrm{P}^{+}$. After implantation, the PLM process was performed as described in Sec. II A. SIMS measured a surface concentration of $\mathrm{P}$ of $2 \times 10^{19}$ atoms $\mathrm{cm}^{-3}$, which is well above the insulator-to-metal transition for $\mathrm{Si}: \mathrm{P}^{59}$ ensuring that no carrier freeze-out would occur under the contacts. E-beam evaporated contacts consisting of a stack of $\mathrm{Ti} / \mathrm{Pd} / \mathrm{Ag}(20 / 20 / 200 \mathrm{~nm})$ defined by photolithography were aligned to the area with the P implant. A schematic of a cross-sectional view of the photoconductivity sample is shown Fig. 2. The control sample with $\mathrm{P}$ implanted everywhere has identical metal contacts and will be referred to as the "contact control sample." A top-down schematic of the photoconductivity sample and the contact control sample are drawn to scale in Fig. 3.

The samples were mounted in a closed-cycle He cryostat for low-temperature measurements. Reported temperatures refer to the actual sample temperature, which was calibrated relative to the cryostat temperature using an independent silicon diode temperature sensor attached to the sample mount. Four-point resistance measurement of the photoconductivity sample as a function of temperature is shown in Fig. 4. The sample resistance increases by several orders of magnitude as the temperature decreases, consistent with carrier freeze-out in the S-doped region. In contrast, the resistance of the contact control sample decreases slightly over the same temperature range, confirming the metallic nature of the $\mathrm{P}$-doped regions. These results confirm two important characteristics of the samples at low temperature. First, the S-doped region is the largest resistor in the photoconductivity sample, and second,

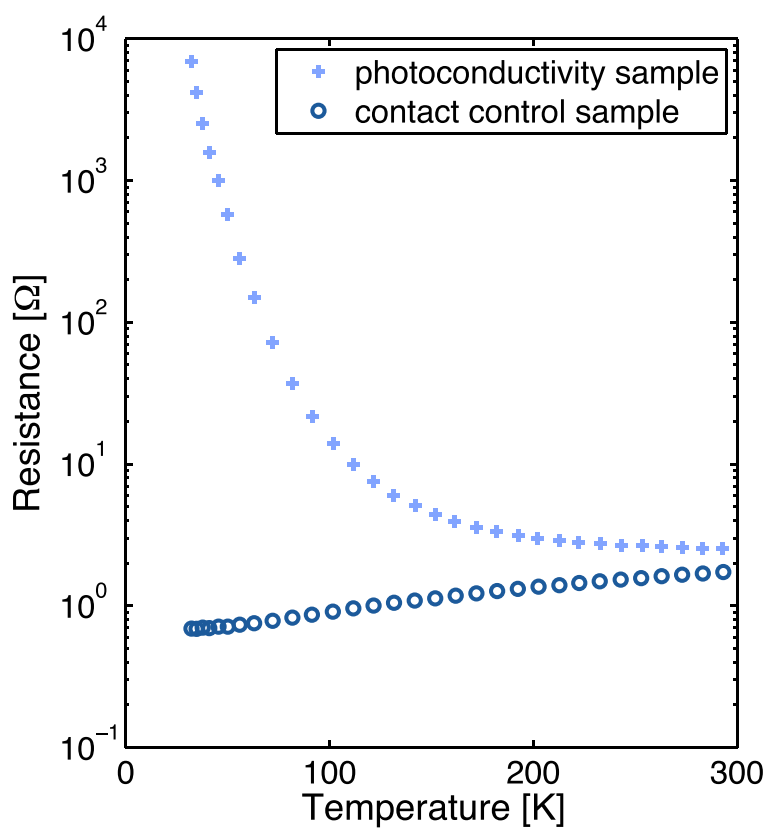

FIG. 4. Sample resistance measured as a function of temperature for the photoconductivity sample and the contact control sample. These data confirm that the S-doped region is the largest resistor in the system at low temperature. 
the resistances of both the metal contact and the regions doped with both $\mathrm{S}$ and $\mathrm{P}$ are negligibly small at low temperature.

A quartz window allowed optical access through the vacuum shroud for sample illumination. Two different monochromatic light sources were used to compare the photo-response with below- and above-band gap illumination. $1550 \mathrm{~nm}$ and $405 \mathrm{~nm}$ laser diodes provided $60 \mathrm{~mW}$ and $0.4 \mathrm{~mW}$ of incident radiation to the sample, respectively. In both cases, the beam spot size on the sample was estimated using various apertures to be below $700 \mu \mathrm{m}$ in diameter, which is smaller than the area enclosed by the busbars and the metal fingers on the sample. The light was mechanically chopped using an optical chopper wheel.

Fig. 2 shows a schematic of the experimental setup for the photoconductivity measurements. A digital sourcemeter provided a DC voltage $V_{\text {app }}$ across the sample and a comparative resistor $R_{\mathrm{C}}$ connected in series. Optical excitation creates excess mobile carriers, causing a drop in the sample resistance upon illumination. The reduction in sample resistance causes an increase in voltage across $R_{\mathrm{C}}$, measured as $\Delta V$. From the circuit in Fig. 2, we can relate $\Delta V$ to $\Delta R$

$$
\frac{\Delta V}{V_{\text {app }}}=\frac{R_{\mathrm{C}}}{R_{\text {avg }}+\frac{\Delta R}{2}+R_{\mathrm{C}}}-\frac{R_{\mathrm{C}}}{R_{\text {avg }}-\frac{\Delta R}{2}+R_{\mathrm{C}}},
$$

where $R_{\text {avg }}$ is the average of the dark and illuminated sample resistances and is determined by $\left.R_{\text {avg }}=\left[\left(V_{\text {app }} / I_{\text {avg }}\right)-R_{\mathrm{C}}\right)\right]$, where $I_{\text {avg }}$ is the average current measured over several cycles. $\Delta V$ is measured as a function of $V_{\text {app }}$ (see Fig. 5(b)), and the slope of this curve gives the right-hand side of Eq. (5). We then solve for $\Delta R$. Additionally, it is clear from Eq. (5) that $\Delta V / V_{\text {app }}$ should peak when $R_{\mathrm{C}}$ is equal to $R_{\text {avg }}$. A plot of $\Delta V / V_{\text {app }}$ for different values of $R_{\mathrm{C}}$ is given in Fig. 5(a); Eq. (5) is used to fit the data. The excellent quality of the fits to the data using a single fitting parameter suggests that the system is well described by the circuit shown in Fig. 2 and that no capacitive or inductive effects in the system impact the measurements.

In Fig. 5(b), $\Delta V$ is plotted against $V_{\text {app }}$ for two different chopping frequencies of the sub-band gap $1550 \mathrm{~nm}$ light. A linear trend is observed in both cases, as is expected from Eq. (5), but the signal changes significantly with chopping frequency. As will be discussed in detail below, the frequency-dependent signal is attributed to sample heating. As seen in Fig. 4, the sample resistance is temperature sensitive, so heating that occurs upon sample illumination can yield a false photoconductive response. To separate the photoconductive signal from any thermal artifacts, the response was measured over a wide range of chopping frequencies. At low chopping frequencies $(<9 \mathrm{~Hz})$, the sample resistance was measured as a function of time using a Keithley 2400 digital sourcemeter, which had a temporal resolution of $40 \mathrm{~ms}$. At high frequencies, a Signal Recovery 7265 lock-in amplifier was used to measure $\Delta V$, as shown in Fig. 2.

\section{B. Experimental results}

Based on the sample geometry and contact pattern, which are represented schematically in Fig. 3, the instantaneous
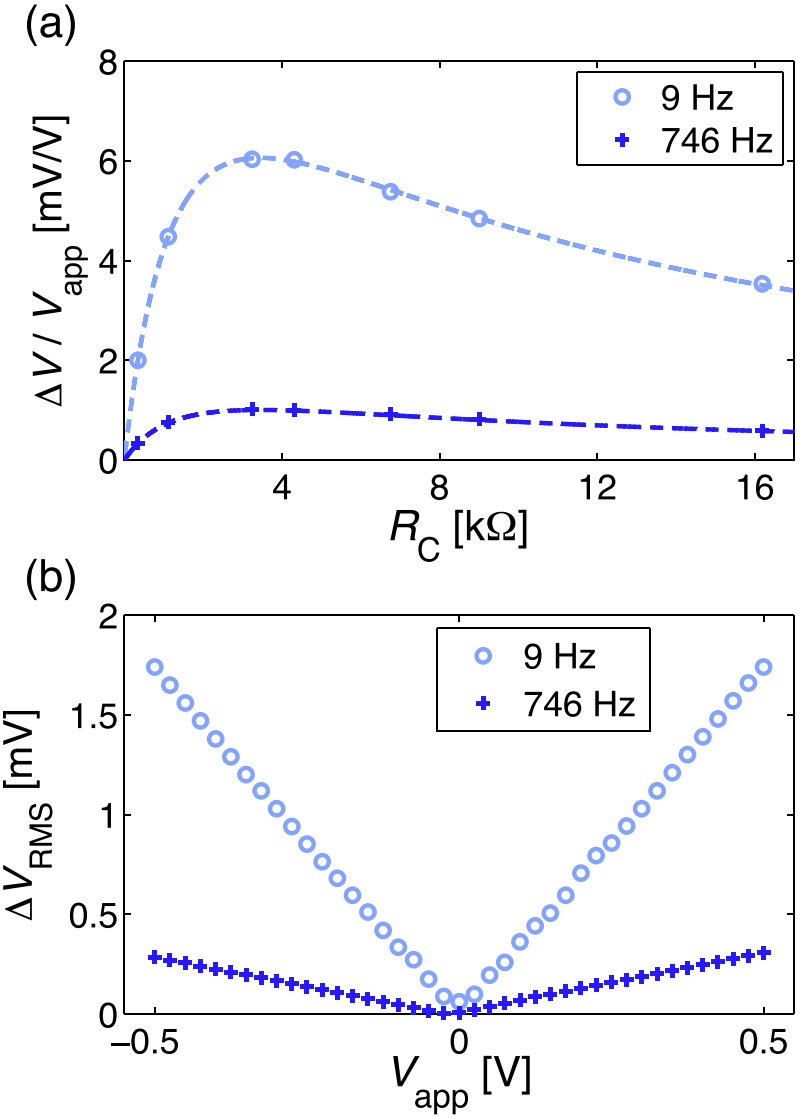

FIG. 5. (a) Ratio of $\Delta V / V_{\text {app }}$ plotted against different comparative resistors, $R_{\mathrm{C}}$, and (b) lock-in voltage, $\Delta V_{\mathrm{RMS}}$, measured as a function of the applied DC bias, $V_{\text {app }}$, for $R_{\mathrm{C}}=3242 \Omega$ at the highest and lowest chopper frequencies measured. Data in both plots were taken with the photoconductivity sample illuminated with $1550 \mathrm{~nm}$ light.

resistance $R$ of the photoconductivity sample is related to the sheet conductivity $\sigma_{\mathrm{s}}$ and the number of fingers protruding from each busbar $N_{\mathrm{f}}$ by Ohm's Law

$$
R=\frac{d}{\sigma_{\mathrm{s}}\left(2 N_{\mathrm{f}}-1\right) L} .
$$

Sheet conductivity of the S-doped region at $36 \mathrm{~K}$, under irradiation by chopped $1550 \mathrm{~nm}$ light, is plotted as a function of time in Fig. 6(a). For the lowest chopping frequency, $0.01 \mathrm{~Hz}$, a rise time of around $10 \mathrm{~s}$ is observed for the conductivity to reach steady state. We attribute this slow response time to heating of the sample surface by the laser. While a purely photoconductive response would reach steady state much faster than the time constant measured here, a thermal response time can be quite slow because it is determined by the thermal mass and thermal diffusivity of both the substrate and the sample mount.

Because the thermally induced response occurs over a time scale many orders of magnitude slower than a photoconductive response, the two competing effects can be separated and individually quantified simply by changing the light-chopping frequency. The measured change in sheet conductivity, $\Delta \sigma_{\mathrm{s}}$, is simply the sum of the photoconductive response and thermal heating response

$$
\Delta \sigma_{\mathrm{s}}=\Delta n_{\mathrm{s}} \mu_{\mathrm{e}} e+\Delta \sigma_{\text {sthermal }} .
$$


(a)
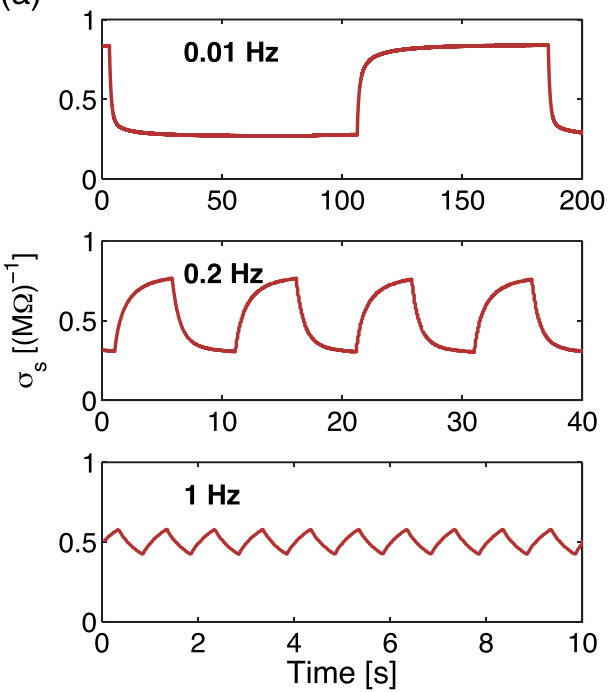

(b)

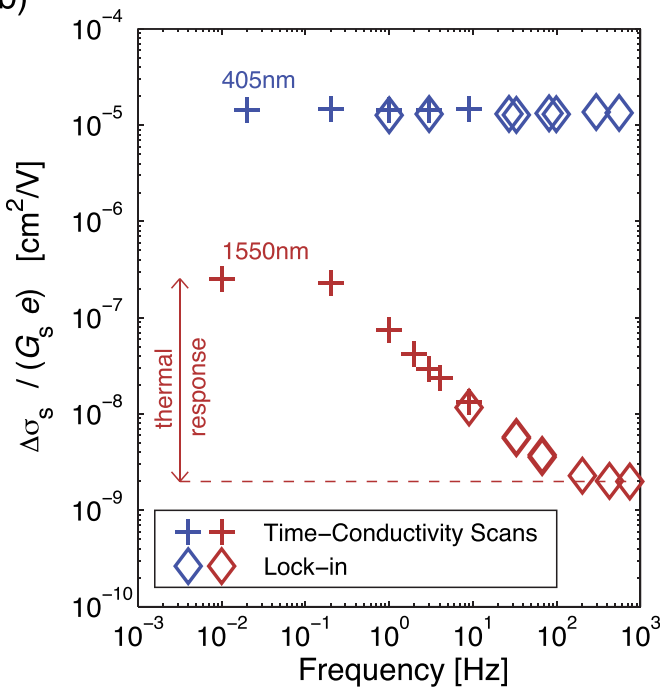

FIG. 6. (a) Sheet conductivity of the S-doped doped region, $\sigma_{\mathrm{s}}$, measured as a function of time under $1550 \mathrm{~nm}$ irradiation chopped at different frequencies. (b) Peak-to-peak change in sheet conductivity divided by the flux of absorbed photons in the sample, $G_{\mathrm{s}}$, and the elementary charge, $e$, plotted against chopper frequency for two different wavelengths of monochromatic illumination ( $405 \mathrm{~nm}$ and $1550 \mathrm{~nm}$ ). Two complementary methods were used to measure the change in sheet conductivity across the frequency range shown: at low frequencies, $\Delta \sigma_{\mathrm{s}}$ was determined from time-resolved conductivity scans (as shown in panel (a)), and at high frequencies, $\Delta \sigma_{\mathrm{s}}$ was measured directly with a lock-in amplifier.

Chopping at frequencies faster than the thermal response time reduces the portion of the AC signal that is derived from sample heating $\left(\Delta \sigma_{\text {sthermal }}\right)$. This effect is seen in Fig. 6(a), where time-conductivity scans reveal that the peak-topeak change in $\sigma_{\mathrm{s}}$ is reduced as the chopping frequency is increased. In Fig. 6(b), the peak-to-peak change in $\sigma_{\mathrm{s}}$ is divided by the product of the sheet carrier generation rate $G_{\mathrm{s}}$ (described below) and the elementary charge $e$ and plotted against chopping frequency. Fig. 6(b) combines measurements done using two complementary techniques: time-conductivity scans at low chopping frequencies and measurements taken using the lock-in amplifier in the circuit shown in Fig. 2. While the sample is illuminated, $\Delta n_{s}$ is determined by a balance between the sheet generation rate $G_{\mathrm{s}}$ and the lifetime of the photo-excited carriers

$$
\Delta n_{\mathrm{s}}=G_{\mathrm{s}} \tau_{\mathrm{e}}
$$

The applicability of this formula is subtle, depending on the electronic state of the traps. We discuss this issue further in Sec. V. We calculate a sheet generation rate, $G_{\mathrm{s}}=N_{\mathrm{ph}}\left(1-R_{\mathrm{m}}-T_{\mathrm{m}}\right) / A$, where $N_{\mathrm{ph}}$ is the rate at which photons hit the sample (measured with a calibrated photodiode), $R_{\mathrm{m}}$ and $T_{\mathrm{m}}$ are the measured transmittance and reflectance, and $A$ is the active area of the device $\left(1.0 \times 1.5 \mathrm{~mm}^{2}\right)$. At high chopping frequencies, the measured photo-response plateaus, as shown in Fig. 6(b). We believe that the plateau occurs when the thermal response, $\Delta \sigma_{\mathrm{s}_{\text {thermal }}}$, is reduced to a value well below that of the true photoconductive response, $\Delta n_{\mathrm{s}} \mu_{\mathrm{e}} e$. Using the value of $\Delta \sigma_{\mathrm{s}} / G_{\mathrm{s}} e$ measured in the highfrequency plateau regime, we can estimate $\mu_{\mathrm{e}} \tau_{\mathrm{e}}$

$$
\mu_{\mathrm{e}} \tau_{\mathrm{e}} \leq\left.\frac{\Delta \sigma_{\mathrm{s}}}{G_{\mathrm{s}} e}\right|_{\text {high freq. }}=(2.5 \pm 1.5) \times 10^{-9} \frac{\mathrm{cm}^{2}}{\mathrm{~V}} .
$$

The value of $2.5 \times 10^{-9} \mathrm{~cm}^{2} / \mathrm{V}$ in Eq. (9) represents an upper limit on the $\mu_{\mathrm{e}} \tau_{\mathrm{e}}$ product because we cannot rule out the possibility that additional artifacts, such as internal photoemission from the contacts, contribute to the response. The value of this sub-band gap response is consistent with the previous contactless measurements, ${ }^{33}$ and provides an upper limit that is nearly two orders of magnitude lower than the previous room temperature measurements. ${ }^{29}$ It is important to note that carrier lifetimes are expected to be much higher at lower temperatures (a decreased thermal velocity $v_{\text {the }_{\mathrm{e}}}$ and decreased capture cross-section ${ }^{60}$ leads to higher lifetimes) and thus the figure of merit at room temperature is expected be lower than the value measured here.

The photoconductivity measurement was repeated using above-band gap light as a control. The response at $405 \mathrm{~nm}$ is plotted as a function of frequency in Fig. 6(b). The 405 nm response is almost two orders of magnitude stronger, and in contrast to the case for sub-band gap illumination, the $405 \mathrm{~nm}$ response is independent of chopping frequency; this is an indication that thermal effects in this case contribute minimally, and that the measured signal is dominated by a purely photoconductive response. The absorption length for $405 \mathrm{~nm}$ light is estimated to be around $500 \mathrm{~nm},{ }^{61}$ which is deeper than the hyperdoped sulfur layer. Therefore, quantitative analysis of the response is complicated by contributions from carriers generated in the silicon substrate, and the measured $\Delta \sigma_{\mathrm{s}} / G_{\mathrm{s}} e$ ratio provides an upper limit of the mobility-lifetime product for the S-doped layer. This complication is not present when $1550 \mathrm{~nm}$ light is used because the sub-band gap wavelength is not absorbed in the substrate and thus does not generate free carriers in the Si substrate.

\section{DISCUSSION}

An IBPV device incorporating deep-level impurities in an absorber layer can have an enhanced efficiency compared to a single junction device as long as the IB material has a 
sufficiently high $\nu$ for both electrons and holes, and meets the other requirements outlined in the Introduction. We have shown through optical measurements that $\mathrm{Si}: \mathrm{S}$ samples with $\mathrm{S}$ concentrations of $2.1 \times 10^{20} \mathrm{~cm}^{-3}$ and below have a measurable optical gap (i.e., a vanishing $\alpha_{\mathrm{e}}$ at zero photon energy) between the impurity states and the conduction band. This indicates that $\mathrm{S}$ concentrations below this value lead to a material with an electronic structure suitable for IBPV. We must then proceed to determine if our material has properties that meet the requirements for a high $\nu$.

Measurements presented here empirically determine $\nu_{\mathrm{e}}$ for Si:S. Combining the optical data and the photoconductivity results, an upper bound on $\nu_{e}$ can be calculated for a sample with a $1.2 \times 10^{20} \mathrm{~cm}^{-3}$ peak S concentration (the lowest dose analyzed here). Low-temperature photoconductivity measurements yield an upper bound on $\mu_{e} \tau_{e}$ of $2.5 \times 10^{-9} \mathrm{~cm}^{2} / \mathrm{V}$ for carriers generated using $1550 \mathrm{~nm}$ light. From Fig. $1, \alpha_{\mathrm{e}}$ is $8300 \mathrm{~cm}^{-1}$ for $1550 \mathrm{~nm}$ photons. Using an upper bound for $V_{\mathrm{bi}}$ of the Si band gap potential of $1.1 \mathrm{~V}$ and a value of $c=2$, an upper limit on the figure of merit described in Eq. (1) is calculated

$$
\nu_{\mathrm{e}} \leq 0.05 .
$$

To make a useful IB absorber layer, $\nu_{\mathrm{e}}$ must be greater than unity; thus, S-doped silicon at a peak concentration of $1.2 \times 10^{20} \mathrm{~cm}^{-3}$ is currently unsuitable for an IB absorber layer, at least as produced by our current fabrication method. We cannot increase $V_{\text {bi }}$ or $\alpha$ for this system, and do not believe that it would be easy to improve the $\mu_{\mathrm{e}} \tau_{\mathrm{e}}$ by a factor of 20. Therefore, we conclude that $\mathrm{Si}: \mathrm{S}$ at a concentration near $10^{20} \mathrm{~cm}^{-3}$ is not a good candidate for IBPV.

Had we observed $\nu_{\mathrm{e}} \gg 1$, then further experimental work would be needed to understand optical cross-section and lifetime of the holes. Additionally, we have only calculated $\nu_{\mathrm{e}}$ at a single photon energy that is near the peak of the absorption spectrum; had $\nu_{\mathrm{e}}$ been above unity, then spectral characterization and proper weighting with the solar spectrum would be necessary to further evaluate the material.

\section{PHOTOCONDUCTIVE DETERMINATION OF TRAP FILLING}

Sulfur is generally found to be a (double) donor in $\mathrm{Si}^{40}$ so it is generally assumed that the $S$ trap states are filled at equilibrium at low temperature. We now show that this assumption is incompatible with the photoconductivity measurements of Sec. III B, and we must have an appreciable $(\approx 10 \%)$ fraction of empty sulfur trap states at equilibrium to explain our results.

If we approximate the IB as being at a single energy (neglecting the dispersion $E_{\sigma}$ of Table I), we can use the classic trapping results of Shockley and Read, ${ }^{62}$ giving

$$
\frac{d \Delta n}{d t}=-\frac{n f_{\mathrm{pt}}-f_{\mathrm{t}} n_{1}}{\tau_{\mathrm{e} 0}}+G,
$$

where $f_{\mathrm{pt}}$ is the fraction of trap states that are empty, $f_{\mathrm{t}}$ is the fraction of trapped states that are filled, and $n_{1}$ is the concentration of occupied conduction band states if the Fermi level is at the trap level, $G$ is the volumetric generation rate, and we have assumed non-degenerate statistics for the conduction band. If we use an effective thickness for the hyperdoped layer of $d=270 \mathrm{~nm}$, then we turn all of our measured sheet values into volumetric values. For example, the volumetric generation rate $G$ is estimated from a sheet generation rate by $G=G_{\mathrm{s}} / d$. The bare trapping rate $1 / \tau_{\mathrm{e} 0}$ is the rate at which an electron is trapped into an entirely empty set of traps and is expressed as $1 / \tau_{\mathrm{e} 0}=N_{\mathrm{T}} v_{\mathrm{th}_{\mathrm{e}}} \sigma_{\mathrm{e}}$, where $v_{\mathrm{th}}$ is the thermal velocity of electrons in the conduction band and $\sigma_{\mathrm{e}}$ is the trapping cross section, found to be $2 \times 10^{-15} \mathrm{~cm}^{2}$ for $\mathrm{S}$ in $\mathrm{Si}$ at low concentrations. ${ }^{63}$ For $N_{\mathrm{T}}=3.8 \times 10^{20} \mathrm{~cm}^{-3}$, we expect $\tau_{\mathrm{e} 0}=2$ ps. Assuming that all excess carriers in the conduction band originate from the trap states (and not through excitation from the VB), we have $\Delta n=N_{\mathrm{T}}\left(f_{\mathrm{pt}}-f_{\mathrm{pt}, 0}\right)$, where $f_{\mathrm{pt}, 0}$ is the fraction of empty trap states at equilibrium.

We can find the steady-state $\Delta n$ by setting Eq. (11) to zero. Since the background $\mathrm{B}$ acceptor concentration is $(3.5 \pm 1.5) \times 10^{14} \mathrm{~cm}^{-3}$, much less than $N_{\mathrm{T}}$, and sulfur is generally a double-donor in $\mathrm{Si}^{40}$ one might assume that at equilibrium the trap states are mostly full $\left(f_{\mathrm{pt}, 0} \approx 0\right)$. As we will describe, we cannot explain our measurements in this case. We demonstrate this result by first analyzing the system in the limit that $f_{\mathrm{pt}, 0}$ is small.

When $f_{\mathrm{pt}, 0}$ is near zero, we have

$$
\Delta n=\frac{1}{2}\left(\sqrt{n_{0}^{2}+4 G \tau_{\mathrm{e} 0} N_{\mathrm{T}}}-n_{0}\right),
$$

where $n_{0}$ is the equilibrium conduction band concentration. If our high-concentration $\mathrm{S}$ behaves similarly to lowconcentration $\mathrm{S}$, we expect $4 G \tau_{\mathrm{e} 0} N_{T}=4 \times 10^{31} \mathrm{~cm}^{-6}$, so $n_{0}$ can be neglected in Eq. (12), resulting in $\Delta n \approx \sqrt{G \tau_{\mathrm{e} 0} N_{\mathrm{T}}}$. Note that this form is different from Eq. (8), and has a quadratic dependence of $\Delta n$ on $G$. Given the values of mobility measured in similar Si:S systems,${ }^{57}$ this should result in $\Delta \sigma_{\mathrm{e}} \approx 0.3-3 \Omega^{-1} \mathrm{~cm}^{-1}$, which is six orders of magnitude larger than the observed signal. Alternatively, the system would require $\tau_{\mathrm{e} 0}$ to be of order $10^{-18} \mathrm{~s}$, faster than thermalization times, to explain the observed conductivity change. We conclude that the system cannot be described with an IB that is nearly completely filled at equilibrium.

When $f_{\mathrm{pt}, 0}$ and $N_{\mathrm{T}}$ are sufficiently large that $f_{\mathrm{pt}}$ is always approximately $f_{\mathrm{pt}, 0}$, we have the simple result

$$
\Delta n=\frac{G \tau_{\mathrm{e} 0}-n_{1}\left(1-f_{\mathrm{pt}, 0}\right)}{f_{\mathrm{pt}, 0}} .
$$

Since we expect $n_{1} \ll G \tau_{\mathrm{e} 0}$, we can neglect the reemission term and find $\Delta n \approx G \tau_{\mathrm{e} 0} / f_{\mathrm{pt}, 0}$. This results in an effective trapping time $\tau_{\mathrm{e}}=\tau_{\mathrm{e} 0} / f_{\mathrm{pt}, 0}$, so $\Delta n=G \tau_{\mathrm{e}}$, which is Eq. (8).

Previous Hall effect measurements have found mobilities of similar Si:S samples to be between 15 and $150 \mathrm{~cm}^{2} / \mathrm{V} \mathrm{s}$ between $36 \mathrm{~K}$ and room temperature. ${ }^{57}$ These values combined with the measured $\mu_{\mathrm{e}} \tau_{\mathrm{e}}$ from Eq. (9) result in $\tau_{\mathrm{e}}$ less than 13-130 ps. These lifetimes are consistent with the hyperdoped $\mathrm{Si}: \mathrm{S}$ samples having a capture cross section 
similar to lightly doped ${ }^{63} \mathrm{Si}: \mathrm{S}$ and $f_{\mathrm{pt}, 0}$ of 0.015 to 0.15 . The current experiments do not explain why such a large fraction of S states are empty at equilibrium. It is unlikely that these states are empty due to thermal excitation to the CB; Hall effect measurements ${ }^{57}$ demonstrate that the ratio of the number of free carriers to the number of $S$ atoms is much less than 0.015 at low temperature. One explanation could be due to non-equilibrium chemical states of S (e.g., interstitials) acting as acceptors at high sulfur concentration. The existence of such a large number of empty $\mathrm{S}$ states at equilibrium is important for understanding and exploiting $\mathrm{Si}: \mathrm{S}$ materials.

\section{CONCLUSIONS}

In this paper, two experiments-FTIR optical spectroscopy and low-temperature photoconductivity-are used in conjunction to evaluate the efficiency potential of $\mathrm{Si}: \mathrm{S}$ as an IB absorber layer material. It is demonstrated that using dopant concentrations above the IMT in S-hyperdoped Si yields a band structure with a negligible IB-CB gap that is unsuitable for IBPV. ${ }^{37}$ Using a S concentration below the IMT, we calculate the figure of merit from Krich et al. ${ }^{36}$ to quantify the candidate material's IBPV potential. For the Si:S system with peak $\mathrm{S}$ concentration of $1.2 \times 10^{20} \mathrm{~cm}^{-3}$, we determine $\nu_{e} \leq 0.05$-more than one order of magnitude too low to create an IBPV device with efficiency greater than a standard silicon device. An estimate for the upper limit of the figure of merit $\nu_{\mathrm{e}}$ suggests that the $\mathrm{Si}: \mathrm{S}$ system is currently unsuitable for IBPV applications.

Additionally, the low-temperature photoconductivity results yield insight into the trap-filling fraction for S-hyperdoped Si. Further experimentation to determine the actual fraction of unoccupied S states will yield insight on how the recombination statistics in hyperdoped materials compares to statistics for isolated defects developed by Shockley and Read. ${ }^{62}$

Investigations continue into other promising impurityband absorber-layer materials. One possible approach to identify suitable candidate materials is to select impurities that have a high optical cross-section but a small electrical capture cross-section for both holes and electrons; these values are found in the literature for dilute concentrations of many elements in $\mathrm{Si}$ and other semiconductors.

\section{ACKNOWLEDGMENTS}

The authors acknowledge M. T. Winkler and J. P. Mailoa for useful discussions about the measurements. P. D. Persans is thanked for insightful discussions concerning artifacts in photoconductivity measurements and possible solutions for their prevention. D.R. was supported by the U.S. Army-ARDEC under Contract No. W15QKN-07-P-0092. A.A. was supported by the U.S. Army Research Office under Contract No. W911NF-12-1-0196. J.J.K. was supported by the Natural Sciences and Engineering Research Council of Canada. Research at MIT was supported by National Science Foundation grant for Energy, Power, and Adaptive Systems under Contract No. ECCS-1102050; and in part by the National Science Foundation (NSF) and the Department of Energy (DOE) under NSF CA No. EEC-1041895. Funding was provided in part by the MIT-KFUPM Center for Clean Water and Energy. This work was performed in part at the Center for Nanoscale Systems (CNS), a member of the National Nanotechnology Infrastructure Network (NNIN), which was supported by the National Science Foundation under NSF Award No. ECS-0335765. CNS is part of Harvard University.

${ }^{1}$ W. Shockley and H. J. Queisser, J. Appl. Phys. 32, 510 (1961).

${ }^{2}$ A. Luque and A. Martí, Phys. Rev. Lett. 78, 5014 (1997).

${ }^{3}$ A. Luque and A. Martí, Prog. Photovoltaics 9, 73 (2001).

${ }^{4}$ A. Luque, A. Martí, E. Antólin, and C. Tablero, Physica B 382, 320 (2006).

${ }^{5}$ G. Conibeer, Mater. Today 10, 42 (2007).

${ }^{6}$ A. Luque, A. Martí, C. Stanley, N. López, L. Cuadra, D. Zhou, J. L. Pearson, and A. Mckee, J. Appl. Phys. 96, 903 (2004).

${ }^{7}$ S. P. Bremner, M. Y. Levy, and C. B. Honsberg, Appl. Phys. Lett. 92, 171110 (2008).

${ }^{8}$ M. Y. Levy and C. Honsberg, Phys. Rev. B 78, 165122 (2008).

${ }^{9}$ K. M. Yu, W. Walukiewicz, J. Wu, W. Shan, J. Beeman, M. Scarpulla, O. Dubon, and P. Becla, Phys. Rev. Lett. 91, 246403 (2003).

${ }^{10}$ N. López, L. Reichertz, K. M. Yu, K. Campman, and W. Walukiewicz, Phys. Rev. Lett. 106, 028701 (2011).

${ }^{11}$ W. Wang, A. S. Lin, J. D. Phillips, and W. K. Metzger, Appl. Phys. Lett. 95, 261107 (2009).

${ }^{12}$ W. Wang, A. S. Lin, and J. D. Phillips, Appl. Phys. Lett. 95, 011103 (2009).

${ }^{13}$ T. Tanaka, K. M. Yu, A. Levander, O. Dubon, L. Reichertz, N. Lopez, M. Nishio, and W. Walukiewicz, Jpn. J. Appl. Phys., Part 1 50, 082304 (2011).

${ }^{14}$ T. Tanaka, M. Miyabara, Y. Nagao, K. Saito, Q. Guo, M. Nishio, K. M. Yu, and W. Walukiewicz, Appl. Phys. Lett. 102, 052111 (2013).

${ }^{15}$ A. Martí, E. Antolin, C. R. Stanley, C. D. Farmer, N. López, P. Diaz, E. Cánovas, P. G. Linares, and A. Luque, Phys. Rev. Lett. 97, 247701 (2006).

${ }^{16}$ A. Martí, N. López, E. Antólin, E. Cánovas, C. Stanley, C. Farmer, L. Cuadra, and A. Luque, Thin Solid Films 511-512, 638 (2006).

${ }^{17}$ E. Cánovas, A. Martí, N. López, E. Antolín, P. Linares, C. Farmer, C. Stanley, and A. Luque, Thin Solid Films 516, 6943 (2008).

${ }^{18}$ A. Luque and A. Martí, Adv. Mater. 22, 160 (2010).

${ }^{19}$ E. García-Hemme, R. García-Hernansanz, J. Olea, D. Pastor, A. del Prado, I. Mártil, and G. González-Díaz, Appl. Phys. Lett. 101, 192101 (2012).

${ }^{20}$ D. Pastor, J. Olea, A. del Prado, E. García-Hemme, R. García-Hernansanz, and G. González-Díaz, Sol. Energy Mater. Sol. Cells 104, 159 (2012).

${ }^{21}$ H. Castán, E. Pérez, H. García, S. Dueñas, L. Bailón, J. Olea, D. Pastor, E. García-Hemme, M. Irigoyen, and G. González-Díaz, J. Appl. Phys. 113, 024104 (2013).

${ }^{22}$ J. Olea, M. Toledano-Luque, D. Pastor, G. Gónzalez-Díaz, and I. Mártil, J. Appl. Phys. 104, 016105 (2008).

${ }^{23}$ J. Olea, M. Toledano-Luque, D. Pastor, E. San-Andres, I. Mártil, and G. Gónzalez-Díaz, J. Appl. Phys. 107, 103524 (2010).

${ }^{24}$ J. Olea, D. Pastor, I. Mártil, and G. González-Díaz, Sol. Energy Mater. Sol. Cells 94, 1907 (2010).

${ }^{25}$ J. Olea, D. Pastor, M. Toledano-Luque, I. Mártil, and G. Gónzalez-Díaz, J. Appl. Phys. 110, 064501 (2011).

${ }^{26}$ G. Gónzalez-Díaz, J. Olea, I. Mártil, D. Pastor, A. Martí, E. Antolín, and A. Luque, Sol. Energy Mater. Sol. Cells 93, 1668 (2009).

${ }^{27}$ B. P. Bob, A. Kohno, S. Charnvanichborikarn, J. M. Warrender, I. Umezu, M. Tabbal, J. S. Williams, and M. J. Aziz, J. Appl. Phys. 107, 123506 (2010).

${ }^{28}$ A. J. Said, D. Recht, J. T. Sullivan, J. M. Warrender, T. Buonassisi, P. D. Persans, and M. J. Aziz, Appl. Phys. Lett. 99, 073503 (2011).

${ }^{29}$ P. D. Persans, N. Berry, D. Recht, D. Hutchinson, A. J. Said, J. M. Warrender, H. Peterson, A. DiFranzo, C. McGahan, J. Clark, W. Cunningham, and M. J. Aziz, Symposium A - Amorphous and Polycrystalline Thin-Film Silicon Science and Technology, edited by B. Yan, S. Higashi, C. C. Tsai, Q. Wang, and H. Gleskova (Mater. Res. Soc. Symp. Proc., 2011), Vol. 1321, p. 291.

${ }^{30}$ J. Sullivan, R. Wilks, M. T. Winkler, L. Weinhardt, D. Recht, A. J. Said, B. Newman, Y. Zhang, M. Blum, and S. Krause, Appl. Phys. Lett. 99, 142102 (2011). 
${ }^{31}$ E. Ertekin, M. T. Winkler, D. Recht, A. J. Said, M. J. Aziz, T. Buonassisi, and J. C. Grossman, Phys. Rev. Lett. 108, 026401 (2012).

${ }^{32}$ D. Recht, J. Sullivan, R. Reedy, T. Buonassisi, and M. J. Aziz, Appl. Phys. Lett. 100, 112112 (2012).

${ }^{33}$ D. Recht, D. Hutchinson, T. Cruson, A. DiFranzo, A. McAllister, A. J. Said, J. Warrender, P. D. Persans, and M. J. Aziz, Appl. Phys. Express 5, 041301 (2012).

${ }^{34}$ P. D. Persans, N. Berry, D. Recht, D. Hutchinson, H. Peterson, J. Clark, S. Charnvanichborikarn, J. Williams, A. DiFranzo, M. J. Aziz, and J. M. Warrender, Appl. Phys. Lett. 101, 111105 (2012).

${ }^{35}$ F. Fabbri, M. J. Smith, D. Recht, M. J. Aziz, S. Gradečak, and G. Salviati, Appl. Phys. Lett. 102, 031909 (2013).

${ }^{36}$ J. J. Krich, B. I. Halperin, and A. Aspuru-Guzik, J. Appl. Phys. 112, 013707 (2012).

${ }^{37}$ A. Luque, A. Martí, E. Antolín, P. G. Linares, I. Tobías, and I. Ramiro, AIP Adv. 1, 022125 (2011).

${ }^{38}$ M. Y. Levy and C. Honsberg, J. Appl. Phys. 106, 073103 (2009).

${ }^{39}$ A. S. Lin, W. Wang, and J. D. Phillips, J. Appl. Phys. 105, 064512 (2009).

${ }^{40}$ E. Janzén, R. Stedman, G. Grossmann, and H. G. Grimmeiss, Phys. Rev. B 29, 1907 (1984).

${ }^{41}$ T. G. Kim, J. M. Warrender, and M. J. Aziz, Appl. Phys. Lett. 88, 241902 (2006).

${ }^{42}$ J. Carey, C. H. Crouch, M. Shen, and E. Mazur, Opt. Lett. 30, 1773 (2005).

${ }^{43}$ M. T. Winkler, D. Recht, M. Sher, A. J. Said, E. Mazur, and M. J. Aziz, Phys. Rev. Lett. 106, 178701 (2011).

${ }^{44}$ M. Green, Silicon Solar Cells: Advanced Principals and Practices (Centre for Photovoltaic Devices and Systems, New South Wales, Australia, 1995).

${ }^{45}$ M. Tabbal, T. Kim, J. Warrender, M. J. Aziz, B. Cardozo, and R. Goldman, J. Vac. Sci. Technol. B 25, 1847 (2007).
${ }^{46}$ See supplementary material at http://dx.doi.org/10.1063/1.4820454 for a SIMS sulfur concentration profile and a detailed description of the model used to determine the real part of the refractive index and $\alpha$ for $\mathrm{Si}: \mathrm{S}$.

${ }^{47}$ S. Pan, D. Recht, S. Charnvanichborikarn, J. Williams, and M. J. Aziz, Appl. Phys. Lett. 98, 121913 (2011).

${ }^{48}$ K. Sánchez, I. Aguilera, P. Palacios, and P. Wahnón, Phys. Rev. B 82, 165201 (2010).

${ }^{49}$ G. Lucovsky, Solid State Commun. 3, 299 (1965).

${ }^{50}$ J. C. Inkson, J. Phys. C 14, 1093 (1981).

${ }^{51}$ L. L. Rosier, J. Appl. Phys. 42, 4000 (1971).

${ }^{52}$ M. Okuyama, N. Matsunaga, J. W. Chen, and A. G. Milnes, J. Electron. Mater. 8, 501 (1979).

${ }^{53}$ M. Jaros, Phys. Rev. B 16, 3694 (1977).

${ }^{54}$ A. Chantre, G. Vincent, and D. Bois, Phys. Rev. B 23, 5335 (1981).

${ }^{55}$ H. G. Grimmeiss and B. Skarstam, Phys. Rev. B 23, 1947 (1981).

${ }^{56}$ H. Pettersson, R. Passler, F. Blaschta, and H. G. Grimmeiss, J. Appl. Phys. 80, 5312 (1996).

${ }^{57} \mathrm{M}$. Winkler, "Non-equilibrium chalcogen concentrations in silicon: Physical structure, electronic transport, and photovoltaic potential," Ph.D. dissertation (Harvard University, 2009).

${ }^{58}$ A. G. U. Perera, H. X. Yuan, and M. H. Francombe, J. Appl. Phys. 77, 915 (1995).

${ }^{59}$ M. Alexander and D. Holcomb, Rev. Mod. Phys. 40, 815 (1968).

${ }^{60}$ C. Henry and D. Lang, Phys. Rev. B 15, 989 (1977).

${ }^{61}$ W. C. Dash and R. Newman, Phys. Rev. 99, 1151 (1955).

${ }^{62}$ W. Shockley and W. T. Read, Phys. Rev. 87, 835 (1952).

${ }^{63}$ H. G. Grimmeiss, E. Janzén, and B. Skarstam, J. Appl. Phys. 51, 4212 (1980). 